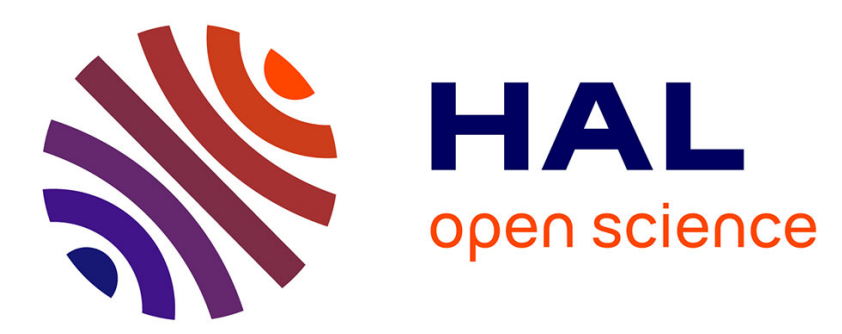

\title{
On the detection of nano dust using spacecraft based boom antennas
}

Filippo Pantellini, G. Le Chat, S. Belheouane, N. Meyer-Vernet, A. Zaslavsky

\section{To cite this version:}

Filippo Pantellini, G. Le Chat, S. Belheouane, N. Meyer-Vernet, A. Zaslavsky. On the detection of nano dust using spacecraft based boom antennas. SOLAR WIND 13: Proceedings of the Thirteenth International Solar Wind Conference, Jun 2012, Big Island, United States. pp.414-417, 10.1063/1.4811073 . hal-03090590

\section{HAL Id: hal-03090590 https://hal.science/hal-03090590}

Submitted on 29 Dec 2020

HAL is a multi-disciplinary open access archive for the deposit and dissemination of scientific research documents, whether they are published or not. The documents may come from teaching and research institutions in France or abroad, or from public or private research centers.
L'archive ouverte pluridisciplinaire HAL, est destinée au dépôt et à la diffusion de documents scientifiques de niveau recherche, publiés ou non, émanant des établissements d'enseignement et de recherche français ou étrangers, des laboratoires publics ou privés. 


\title{
On the detection of nano dust using spacecraft based boom antennas
}

\author{
F. Pantellini*, G. Le Chat ${ }^{\dagger, *}$, S. Belheouane*, N. Meyer-Vernet* and A. Zaslavsky* \\ ${ }^{*}$ LESIA, Observatoire de Paris, CNRS, UPMC, Université Paris Diderot; \\ 5 Place Jules Janssen, 92195 Meudon, France \\ ${ }^{\dagger}$ Harvard-Smithsonian Center for Astrophysics, Cambridge, MA, USA
}

\begin{abstract}
High rate sampling detectors measuring the potential difference between the main body and the boom antennas of interplanetary spacecraft have been shown to be an efficient means to detect impacts of small dust grains in the nanometer size range (see Meyer-Vernet et al., Solar Physics, 256, 463-474, 2009 and Zaslavsky et al., J. Geophys. Res., 117, 05102, 2012). Rough estimates of the free charge $Q$ in the post impact generated plasma cloud indicate that the cloud's own internal electrostatic field is far too weak to rise the antenna's potential by the observed values. We solve this issue by showing that the cloud's internal field is nevertheless strong enough to transitionally interrupt the photoelectron return current towards the portion of the antenna finding itself within the a critical distance $R_{\mathrm{C}} \propto Q^{1 / 3}$ from the impact point. The antenna then steadily increases its positive charge (and its electrostatic potential with respect to the satellite's main body) during a time interval of the order of the inverse of the photoelectron plasma frequency. In previous works we interpreted $R_{\mathrm{C}}$ as the final radius of the expanding cloud. Here we propose that $R_{\mathrm{C}}$ is the distance beyond which the cloud's field is efficiently screened by the ambient electrons.
\end{abstract}

Keywords: Interplanetary dust, plasma interaction with antennas, photoemission PACS: $96.50 . \mathrm{Dj}, 52.40 . \mathrm{Fd}, 79.60 . \mathrm{Ht}, 79.60 . \mathrm{Ht}$

\section{INTRODUCTION}

Following a nano dust grain impact on the spacecraft body a dense plasma cloud with a free charge of the order $Q \sim 310^{-12} \mathrm{C}[1,2]$ expands around the impact point (see Fig. 2a,b). For dust grains in the nanometer size domain the cloud's electrostatic field has decreased below the spacecraft's own field $E_{\mathrm{SC}}$ when reaching a $\mathrm{cm}$ order radius. The cloud's electrons are then rapidly recollected by the spacecraft leaving a pure ion cloud pursue expansion (Fig. 2c). The recollected electrons decrease the spacecraft's body charge by $-Q$ leading to a reduction of its potential with respect to other parts of the satellite electrically disconnected from the main body (e.g. the boom antennas). The instrumentally measured potential pulse $\delta V_{\mathrm{SC}}$ is related to the free charge $Q$ and the spacecraft body capacitance $C_{\mathrm{SC}}$ via

$$
\delta V_{\mathrm{SC}}=\Gamma \frac{Q}{C_{\mathrm{SC}}}
$$

where $\Gamma$ is an order unity gain factor. Such impulsive variations of the spacecraft's body potential are indeed measured by the high time resolution TDS (Time Domain Sampler) instrument on STEREO which samples the potential between three mutually orthogonal boom antennas and the spacecraft body. In most cases [3], the detected voltage pulses are not of equal amplitude on the three antennas with one antenna presenting a much stronger signal as illustrated by the characteristic example of Fig. 1.

Estimates of the field intensity around the cloud using Coulomb's law for a $Q$ point charge indicate that the field is far too small to boost the antenna's potential to the observed high levels (like the level of the $x$ antenna in Fig. 1). On the other hand the field turns out to be large enough to substantially increase the angular momentum of a majority of photoelectrons, effectively reducing the photoelectron return current. This occurs because the antenna radius $r_{0}(=1 \mathrm{~cm}$ on STEREO $)$ is much smaller than the typical radius of the photoelectron cloud surrounding the antenna, i.e. much smaller that the photoelectron Debye length $\lambda_{\mathrm{ph}} \sim 1 \mathrm{~m}$ at $1 \mathrm{AU}$ [2]. The smallness of the ratio $r_{0} / \lambda_{\text {ph }}$ implies that most photoelectrons move on high excentricity orbits which can be easily disconnected from the antenna (Fig. 2c). The transitional interruption of the photoelectron return current induces a positive charging of the antenna and a measurable variation of the electrostatic potential difference $\delta V_{\mathrm{a}}$ between the spacecraft and the one antenna exposed to the cloud's field.

In $[3,2]$ it has been argued that the "critical" radius $R_{\mathrm{C}}$ (see equation 2 ), which is a rough estimate of the maximum extension of the cloud's field has to do with the its maximum expansion before dilution in the ambient plasma. In this work we point out that $R_{\mathrm{C}}$ does more pertinently represent the distance beyond which the cloud's field is efficiently screened by the ambient electrons. 


\section{STEREO-TDS OBSERVATIONS}

Typical temporal voltage profiles measured by TDS on the three boom antennas after a nano dust impact is shown in Fig. 1. Dust impact profiles are characterized by a rapid rise phase on a time scale of the order of a few tens of $\mu \mathrm{s}$ [3] followed by a slow recovery phase on $\mathrm{ms}$ time scale. The recovery phase has been shown to be given by the discharge time $R C_{\mathrm{a}}$, where $C_{\mathrm{a}} \approx 60 \mathrm{pF}$ is the antenna capacitance and $R=\left(d I_{\mathrm{ph}} / d \phi_{\mathrm{a}}\right)^{-1}$ is the effective resistance of the antenna at a potential $\phi_{\mathrm{a}}$ subject to a photoelectron current $I_{\mathrm{ph}}$ [4]. Tens of thousands pulses of this type have been detected on STEREO. While for large grains in the $\mu \mathrm{m}$ size domain the amplitudes are approximately equal on the three antennas, smaller, nm size grains are generally characterized by a strong pulse on one antenna and two, roughly equal but much smaller pulses, on the other antennas.
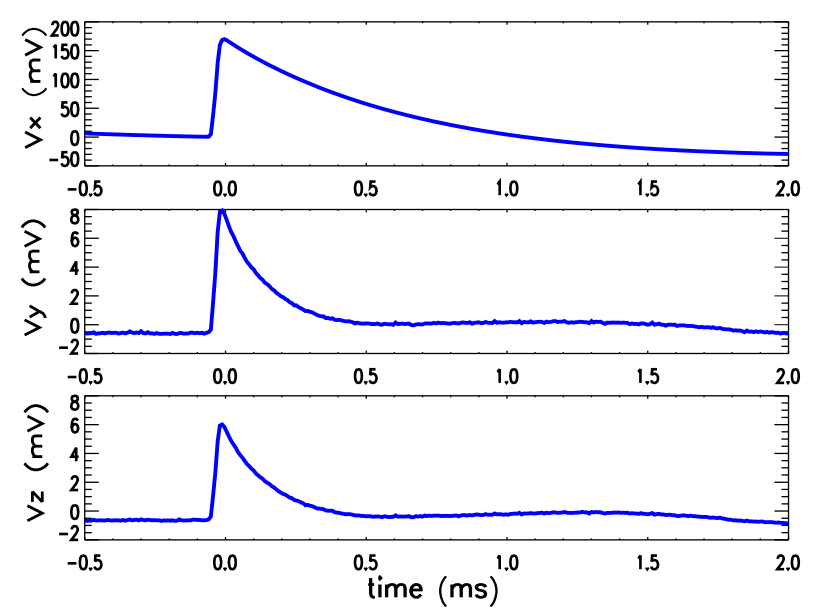

FIGURE 1. Example of voltage pulses detected by TDS on each of the three boom antennas on the STEREO spacecraft following the impact of a nano meter dust particle on the main body of the spacecraft. Note that the pulse is substantially stronger on the $x$-antenna compared to both the $y$ and $z$ antennas.

In the simplest imaginable scenario a dust particle impacting on the spacecraft body (Fig. 2a) generates an expanding plasma cloud as shown in Fig. 2b. Electrons from the cloud are rapidly recollected by the spacecraft's electrostatic field $E_{\mathrm{SC}}$ adding a negative charge $-Q$ to the spacecraft body (Fig. 2c). In small clouds generated by nano dust impacts, the electric field decreases below the spacecraft's field early during the expansion when the cloud's radius is smaller than $10 \mathrm{~cm}$ implying complete recollection of the cloud's electrons on $\mu$ s time scale[2]. The accumulation of charges on the spacecraft body modifies its potential with respect to the conductively disconnected antenna (Fig. 2c). The electrostatic potential between the spacecraft body and the boom antenna suddenly increases by $\delta V_{\mathrm{SC}}$ (cf Eq. (1)). In this simple scenario the potential profile should be similar on all antennas and provide a direct measure of the free charge produced by the impact. This is indeed the case for a small but significant fraction of the impacts detected on STEREO identified as $\mu \mathrm{m}$ grains impacts. However, most of the detected events are characterized by a strong peak on one antenna and two substantially smaller peaks on the other two antennas as in the example of Fig. 1. These are identified as nm grains impacts.

In appendix A of Zaslavsky et al [3] it is suggested that the strong peak occurs on the antenna which has a portion of length $l$ within the expanding plasma cloud at the time of its largest expansion, i.e. at the time when its density has decreased to the level of the ambient density $n_{\mathrm{a}}$ and its radius grown to $R_{\mathrm{C}}$ :

$$
R_{\mathrm{C}}^{3}=\frac{3 Q}{4 \pi n_{\mathrm{a}}} \propto \delta V_{\mathrm{SC}} .
$$

As we shall in the next section $R_{\mathrm{C}}$ should rather be viewed as the maximum extension of the electrostatic potential of the cloud due to screening rather than its maximum physical extension in terms of e.g. ion density. For a given ambient plasma density $n_{\mathrm{a}}$, equation (1) provides a direct relation between the cloud radius $R_{\mathrm{C}}$ and $\delta V_{\mathrm{SC}}$. Zaslavsky et al then make the ansatz that the peak amplitude of the potential on the antenna with the strongest signal is

$$
\delta V_{\mathrm{a}}=\Gamma \frac{k_{\mathrm{B}} T}{e} \frac{l}{L}
$$

where $e$ is the elementary charge, $k_{\mathrm{B}}$ the Boltzmann constant, $L$ the total length of the antenna and $T$ a free parameter with the dimension of temperature. For an impact at a distance $r$ from the base of the antenna, the length $l$ in equation (3) is just $l=\left(R_{\mathrm{C}}^{2}-r^{2}\right)^{1 / 2}$ (see Fig. 2). Assuming dust grains are distributed uniformly in space, the impact probability must increase linearly with $r$ up to a maximum distance $R_{\max }$ (of the order of the linear size of the spacecraft body) beyond which no impacts can occur. The expectation for $\delta V_{\mathrm{a}}$ is then given by [3]

$$
\left\langle\delta V_{\mathrm{a}}\right\rangle= \begin{cases}\alpha T R_{\mathrm{C}}^{3} & , R_{\mathrm{C}} \leq R_{\max } \\ \alpha T R_{\mathrm{C}}^{3}\left[1-\left(1-\frac{R_{\max }^{2}}{R_{\mathrm{C}}^{2}}\right)^{3 / 2}\right] & , R_{\mathrm{C}}>R_{\max }\end{cases}
$$

where $\alpha \equiv 2 \Gamma /\left(3 L R_{\max }^{2}\right)$. Equations (4) and (2) do imply the linear relation $\delta V_{\mathrm{a}} \propto T \delta V_{\mathrm{SC}}$ for clouds with $R_{\mathrm{C}} \leq$ $R_{\text {max }}$. For larger clouds $R_{\mathrm{C}}>R_{\max }$ the relation between $\delta V_{\mathrm{a}}$ and $\delta V_{\mathrm{SC}}$ becomes sub linear allowing for an experimental determination of $R_{\max }$. Direct measurements of $\delta V_{\mathrm{a}}$ and $\delta V_{\mathrm{SC}}$ as reported on Figure A.10 of [3] do indeed show that equation (4) does a remarkable work in fitting the measurements setting $\Gamma=0.5, n_{\mathrm{a}}=5 \mathrm{~cm}^{-3}$ (a 
typical value at $1 \mathrm{AU}), R_{\max } \approx 0.85 \mathrm{~m}$, and a temperature $T$ of the order 2.2 to $2.5 \mathrm{eV}$. Quite remarkably, an excellent fit of the measurements could be obtained with only two free parameters $R_{\max }$ and $T$. A physical interpretation of the empirical law (4) is presented in the next section.

\section{PHYSICAL MECHANISM}

The statistical analysis of the dust impact associated voltage pulses from STEREO-TDS shows that the pulse amplitudes do only depend on $R_{\mathrm{C}}$, or, equivalently on the free charge $Q$, and an ad hoc temperature $T$ in the range 2.2 to $2.5 \mathrm{eV}$. Incidentally, at $1 \mathrm{AU}$, photoelectrons around spacecraft have temperatures of this order [4] suggesting that photoelectrons are a key ingredient in any viable scenario describing the origin of the strong voltage pulse on the $x$-antenna on Fig. 1 . The very fact that the voltage pulses are intimately related to photoelectrons is one reason for ruling out the possibility that the pulses are a direct measure of the electrostatic potential structure of the cloud itself. The other reason is that the electrostatic potential of a cloud generated by the impact of a nano dust is orders of magnitude too weak to rise the antenna's potential to the observed values [2].

Having established that the voltage pulse is due to a perturbation of the photoelectron cloud surrounding the antenna as illustrated in Fig. 2c we make a quantitative estimate of the amplitude of the voltage pulse which is expected if a portion of the antenna of length $l$ does become disconnected from its photoelectrons (which no longer fall back onto the antenna's surface) during the pulse rise time $\tau$. Measurements from TDS on STEREO indicate that $\tau$ is typically of the order of 10 to $80 \mu$ s with a marked peak around $40 \mu \mathrm{s}$.

At $1 \mathrm{AU}$, the charge of photoelectrons emitted during a time $\tau$ by a boom of length $l$, radius $r_{0}$ and oriented perpendicularly to the Sun-satellite direction is of the order

$$
Q_{\mathrm{a}}(l) \sim j_{\mathrm{ph}, 1 \mathrm{AU}} 2 r_{0} l \tau
$$

where $j_{\mathrm{ph}, 1 \mathrm{AU}}=510^{-5} \mathrm{~A} / \mathrm{m}^{2}$. Thus, if as a result of the presence of the dust impact generated cloud the emitted photoelectrons are temporarily unable to fall back over a portion of the antenna a positive charge $Q_{\mathrm{a}}(l)$ must accumulate on its surface. The measured potential of the antenna then rises by an amount $\delta V_{\text {a }}$ proportional to the emitted charge times the inverse of the antenna capacitance $C_{\mathrm{a}}(\approx 60 \mathrm{pF}$ on STEREO)

$$
\delta V_{\mathrm{a}}=\Gamma \frac{Q_{\mathrm{a}}(l)}{C_{\mathrm{a}}} .
$$

Given a typical charging time $\tau=40 \mu$ s we obtain $Q / l=$ $410^{-11} \mathrm{C} / \mathrm{m}$ from (5) and with $\Gamma=0.5$ a voltage pulse of the order $\delta V_{\mathrm{a}} / l=0.32 \mathrm{~V} / \mathrm{m}$. Typical clouds for nano meter sized dust grain impacts extend to $R_{\mathrm{C}} \lesssim 1 \mathrm{~m}$. Assuming $l=0.5 m$ the voltage pulse expected based on (6) is $\delta V_{\mathrm{a}}=0.16 \mathrm{~V}$ a value compatible with the strongest events reported in [3].

Not surprisingly, equation (6) shows that the ansatz (3) rests on the assumption of a rough equipartition between the average potential energy of the photoelectrons bound to a boom antenna of length $l$ (and total charge $Q$ ) and their average thermal energy, viz.

$$
e \frac{Q / l}{C_{\mathrm{a}} / L}=k_{\mathrm{B}} T
$$

At this stage three questions remain open: (1) how do the photoelectrons become disconnected from the antenna despite the smallness of the cloud's internal electrostatic field. (2) What is the meaning of $R_{\mathrm{C}}$ ? (3) What determines the charging time $\tau$.

Concerning question (1). In order to appreciate how small the cloud's internal field can be, let us assume the extreme case of a cloud with all electrons stripped off by the spacecraft's field (cf Fig. 2c). The total free charge in a cloud due to a dust grain of $10^{-20} \mathrm{~kg}$ hitting the spacecraft at $400 \mathrm{~km} / \mathrm{s}$ is of the order of $Q=310^{-12} \mathrm{C}$. For a spherical cloud we can then use the Coulomb expression $Q / 4 \pi \varepsilon_{0} R_{\mathrm{C}}$ to compute the potential difference between $R_{\mathrm{C}}$ and infinity. Now, for $R_{\mathrm{C}}=1 \mathrm{~m}$ the potential difference is down to a ridiculous $0.027 \mathrm{~V}$, clearly insufficient to substantially alter the energy of a typical $\sim 2 \mathrm{eV}$ photoelectron. As we shall see, the field is nevertheless strong enough to prevent photoelectrons emitted by the antenna to be recollected by the same antenna after a ballistic flight, the standard scenario under stationary conditions (Fig. 1). The reason for most photoelectrons being emitted and recollected by the same antenna is that this is the only way to compensate for the strong current of photoelectrons emitted by the sunlit antenna, the other sources of electrons, and particularly the solar wind being unable to provide the necessary flux at $1 \mathrm{AU}$. The key point in our answer to question (1) is that the cloud of photoelectrons around the boom antenna extends to meter order distances from the antenna [2] implying high excentricity orbits for most photoelectrons. Under such circumstances conservation of the angular momentum $M=m r v_{\theta}\left(v_{\theta}\right.$ being the azimuthal velocity component in cylindrical coordinates ) implies that the transverse velocity component $v_{\theta}(r)=v_{\theta}\left(r_{0}\right) r_{0} / r$ is smaller by a factor 10 to 100 over most of its trajectory which spans from the antenna's surface at $r_{0}=1 \mathrm{~cm}$ out to a distance of the order of the photoelectron Debye length $\lambda_{\mathrm{D}} \sim 1 \mathrm{~m}$ [2]. The average $v_{\theta}$ of a photoelectron along its trajectory being a factor $r_{0} / \lambda_{\mathrm{D}} \ll 1$ smaller than at $r=r_{0}$ and smaller by a similar factor with respect to the average radial velocity $v_{r}$, a minor energy kick from the cloud's electrostatic field may suffice to change $v_{\theta}$ by a factor two or 
(a)

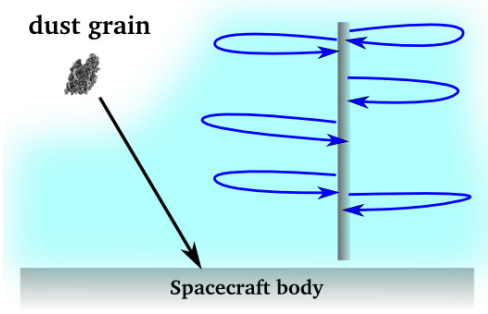

(b)

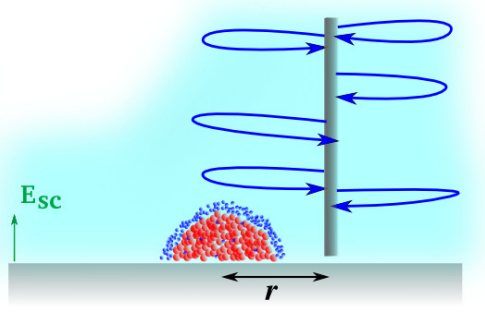

(c)

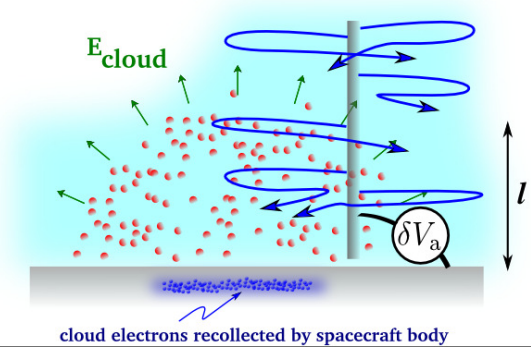

FIGURE 2. Following a dust impact on the main body of a spacecraft (a) a plasma cloud made of ions and electrons expands radially from the impact point (b) at some distance $r$ from a boom antenna. Early during the expansion, the cloud electrons are recollected by the spacecraft under the effect of the spacecraft's own electrostatic field $E_{\mathrm{SC}}$ (c). The cloud's electric field is strong enough to modify the photoelectrons moving on high excentricity orbits so that they are no longer collected by the antenna. During this time the antenna increases its positive charge inducing rising its voltage by $\delta V_{\mathrm{a}}$ with respect to the spacecraft's body.

more, possibly enough to disconnect the trajectory from the antenna surface. The required energy kick to make a photoelectron become disconnected from the antenna can be evaluated to be of the order of the typical energy of a photoelectron $(\sim 2 \mathrm{eV})$ multiplied by the small ratio $\left(r_{0} / \lambda_{\mathrm{D}}\right)^{2}$. From the above discussion we obtain $\left(r_{0} / \lambda_{\mathrm{D}}\right)^{2}$ in the range $10^{-4}$ to $10^{-2}$ and a minimum energy kick in the range $210^{-4}$ to $210^{-2} \mathrm{eV}$ which is much less than the above estimate of $0.027 \mathrm{~V}$ for the cloud's field at $R_{\mathrm{C}}$.

Let us now come to question (2) and the interpretation of the critical radius $R_{\mathrm{C}}$. As already stated above, a few $\mu$ s after the impact essentially all cloud electrons have been recollected by the spacecraft. At that time its radius is of the order of a couple of $\mathrm{cm}$ and the ambient electrons do immediately start moving towards the positive remnant. The number of ambient electrons in a sphere of radius $1.26 R_{\mathrm{C}}<\lambda_{\mathrm{D}}$ is sufficient to completely screen the charge on a short time scale $R_{\mathrm{C}} / v_{\mathrm{e}}$, where $v_{\mathrm{e}}=\left(k_{\mathrm{B}} T / m_{\mathrm{e}}\right)^{1 / 2}$ is the electron thermal velocity. For sub-Debye values of $R_{\mathrm{C}}$ the oscillations associated with the screening are strongly Landau damped and the electric field beyond $R_{\mathrm{C}}$ immediately averaged to zero. The screening process is a fast process involving the spatial scale $R_{\mathrm{C}}$ and the electron thermal velocity. It is sufficiently fast to account for the short time scale of the growth phase in the potential profiles (see Fig. 1). On the other hand, the cloud expansion invoked in $[3,2]$ is a slow process involving $R_{\mathrm{C}}$ and the cloud expansion velocity which is of the order the ion thermal velocity [5]. The associated time scale may be short enough to account for the rapid growth of the potential profiles of Fig. 1 but is definitely slower than screening. In addition, if no screening is invoked there is no reason for the electric field of the cloud to be confined within $R_{\mathrm{C}}$ arising the question of why only the portion of antenna within $R_{\mathrm{C}}$ appears to be affected by the cloud.

Let us now address question (3) on the characteristic charging time $\tau$ in equation (5). According to the above discussion, the charging starts as soon as the cloud's electrons have been recollected by the spacecraft body, i.e. after a few $\mu$ s. Charging stops as soon as the photoelectrons have reestablished a static field around the antenna. The typical time scale for this process is of the order of the inverse photoelectron plasma frequency $2 \pi / \omega_{\mathrm{ph}} \approx$ $10 \mu \mathrm{s}$ (for a reasonable average density $n_{\mathrm{ph}}=100 \mathrm{~cm}^{-3}$ for the photoelectron population around the antenna [2]).

\section{CONCLUSIONS}

We have presented a revised model of the dust impact associated voltage pulses detected by the TDS instrument on STEREO. In the new scenario the critical radius $R_{\mathrm{C}}$ (cf equation 2) is no longer interpreted as the maximum expansion of the impact generated plasma cloud as first proposed in $[3,2]$ but rather as the radius at which the cloud's positive charge is efficiently screened by the ambient electrons.

\section{REFERENCES}

1. N. Meyer-Vernet, M. Maksimovic, A. Czechowski, I. Mann, I. Zouganelis, K. Goetz, M. L. Kaiser, O. C. S. Cyr, J.-L. Bougeret, and S. D. Bale, Solar Physics 256, 463-474 (2009).

2. F. Pantellini, S. Belheouane, N. Meyer-Vernet, and A. Zaslavsky, Astrophysics and Space Science (2012), ISSN 0004-640X, 1572-946X.

3. A. Zaslavsky, N. Meyer-Vernet, I. Mann, A. Czechowski, K. Issautier, G. Le Chat, F. Pantellini, K. Goetz, M. Maksimovic, S. D. Bale, and J. C. Kasper, Journal of Geophysical Research (Space Physics) 117, 05102 (2012).

4. P. Henri, N. Meyer-Vernet, C. Briand, and S. Donato, Physics of Plasmas 18, 2308 (2011).

5. F. Pantellini, S. Landi, A. Zaslavsky, and N. Meyer-Vernet, Plasma Physics and Controlled Fusion 54, 045005 (2012). 